\title{
A VCSEL based system for on-site monitoring of low level methane emission
}

\author{
A. Kannath ${ }^{*}$ a, b, J. Hodgkinson ${ }^{\text {a }}$, R. G. Gillard ${ }^{\text {b }}$ R. J. Riley ${ }^{\text {b }}$, R. P. Tatam ${ }^{\text {a }}$ \\ ${ }^{a}$ Department of Engineering Photonics, School of Engineering, Cranfield University \\ Bedfordshire, MK43 0AL, UK \\ ${ }^{\mathrm{b}}$ Geotechnical Instruments Ltd, Leamington Spa, CV31 3JR, UK
}

\begin{abstract}
Continuous monitoring of methane emissions has assumed greater significance in the recent past due to increasing focus on global warming issues. Many industries have also identified the need for ppm level methane measurement as a means of gaining carbon credits. Conventional instruments based on NDIR spectroscopy are unable to offer the high selectivity and sensitivity required for such measurements. Here we discuss the development of a robust VCSEL based system for accurate low level measurements of methane. A possible area of application is the measurement of residual methane whilst monitoring the output of flare stacks and exhaust gases from methane combustion engines. The system employs a Wavelength Modulation Spectroscopy (WMS) scheme with second harmonic detection at $1651 \mathrm{~nm}$. Optimum modulation frequency and ramp rates were chosen to maintain high resolution and fast response times which are vital for the intended application. Advanced data processing techniques were used to achieve long term sensitivity of the order of $10^{-5}$ in absorbance. The system is immune to cross interference from other gases and its inherent design features makes it ideal for large scale commercial production. The instrument maintains its calibration and offers a completely automated continuous monitoring solution for remote on site deployment.
\end{abstract}

Keywords: Wavelength modulation spectroscopy, VCSEL, residual methane, tuneable diode lasers

\section{INTRODUCTION}

Methane is a major greenhouse gas (GHG) and constitutes around $50-60 \%{ }^{1}$ of landfill gas emission. It has a warming potential over 20 times that of $\mathrm{CO}_{2}$ and has hence received tremendous attention recently in relation to global warming and climate change. Under the Kyoto Protocol ${ }^{2}$ Clean Development Mechanism (CDM), methane can now be traded for carbon credits. Many industries have hence felt the need to accurately measure low ppm levels of residual methane in stack monitoring and also at the output of combustion engines which are needed to burn unused methane. However, conventional instruments based on broadband Non-Dispersive Infrared (NDIR) technology do not offer the high selectivity and sensitivity needed for such precise measurements. This is a specific requirement when targeting the carbon credits market. Tuneable diode laser spectroscopy (TDLS) offers several advantages over conventional NDIR techniques such as high specificity and sensitivity, lower detection limits, auditable function over its lifetime and quick response times of a few seconds. As a consequence, this technique is gaining increasing popularity for gas monitoring in harsh industrial environments ${ }^{3-6}$ and also for trace gas detection in the atmosphere ${ }^{7,8}$.

With the advent of commercially available VCSELs, dependence on the more expensive Distributed Feedback (DFB) laser diodes for TDLS systems has reduced. VCSELs, which initially catered to the telecommunications market, are now being developed to address gas sensing applications in the near infra red. Although a VCSEL has lower output power, it has several advantages over a DFB such as wider tunability, lower power consumption, higher modulation capability and lower cost. In our system we have used a VCSEL with a centre wavelength at $1651 \mathrm{~nm}$ and a tuning range of approximately $4 \mathrm{~nm}$. This makes it possible to probe the R4 quadruplet line of the $2 v_{3}$ absorption band of methane centred on $1.66 \mu \mathrm{m}$. The following sections describe in detail the various steps taken towards the realisation of a VCSEL based methane analyser that is robust and field deployable and provides a solution for automated continuous on site monitoring of the gas.

*a.kannath@geotech.co.uk; phone +44 (0)1926 331068; geotech.co.uk

Vertical-Cavity Surface-Emitting Lasers XV, edited by James K. Guenter, Chun Lei,

Proc. of SPIE Vol. 7952, 79520F - @ 2011 SPIE · CCC code: 0277-786X/11/\$18 · doi: 10.1117/12.874513

Proc. of SPIE Vol. $795279520 \mathrm{~F}-1$ 


\section{OVERVIEW OF WAVELENGTH MODULATION SPECTROSCOPY}

Tuneable diode lasers have very narrow line widths of a few $\mathrm{MHz}$, when compared to a pressure broadened gas line width of a few $\mathrm{GHz}$ under ambient conditions. By controlling the laser drive current and temperature, it is possible to tune the emission wavelength of the laser to match a single absorption line of the target methane gas as shown in figure 1 , thereby eliminating cross gas effects.

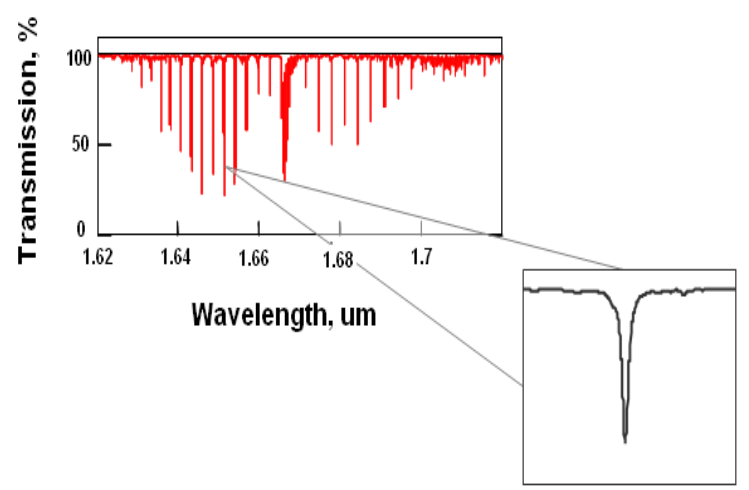

Figure 1. Absorption spectrum of methane showing the $2 v_{3}$ band at $1.66 \mu \mathrm{m}$ with the inset showing the R4 quadruplet gas line at 1651 $\mathrm{nm}$. Generated with data from the HITRAN 2004 database $^{9}$.

In Direct Absorption (DA) spectroscopy, a saw tooth or triangular waveform of low frequency (hundreds of $\mathrm{Hz}$ or a few $\mathrm{kHz}$ ) is added to the diode injection current so that the laser emission wavelength can be made to repeatedly scan across the gas absorption line. A portion of the light is absorbed by the target gas as governed by the Beer Lambert law given by

$$
I=I_{0} \cdot e^{-\sigma(v) c l}
$$

where $I$ is the transmitted intensity, $I_{0}$ is the incident intensity, $\sigma(v)$ is the wavelength dependant absorption cross-section, $c$ is the concentration and $l$ is the absorption path length. The transmitted light is collected by a detector, giving an output with a sloping background as shown in figure 2 . This is because the triangular waveform has the undesirable effect of ramping up the laser output intensity. The disadvantages of the DA technique are the strongly sloping background signal, which makes it difficult to identify small absorptions and the vulnerability of the scheme to small drifts in the laser output power. The detection limit is normally $10^{-3}-10^{-4}$ in terms of absorbance and is limited by baseline instability caused by interference and unstable laser operation.

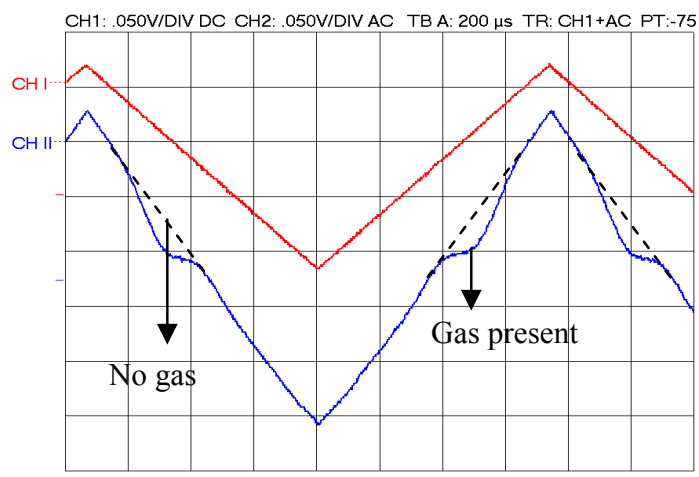

Figure 2. Direct absorption measurement showing the sloping background and the dip in the ramp due to gas absorption in the lower trace. The upper trace is the triangular modulation added to the laser injection current.

In WMS, the slow wavelength scanning of the laser output is combined with a high frequency $\mathrm{F}$ (several $\mathrm{kHz}$ ) low amplitude sine wave modulation of the laser injection current. The interaction of this modulated radiation with the target gas line generates different harmonics of the fundamental modulation frequency which are then recovered using a lock-in amplifier or a phase sensitive detector (PSD). This way, the signal detection is carried out at a much higher frequency, 
reducing $1 / \mathrm{F}$ noise and thereby improving limit of detection to around $10^{-6}$ in absorbance. The resultant first, second and third harmonics of the modulation frequency are shown in figure 3 . As seen in the figure, the second harmonic $2 \mathrm{~F}$ signal peaks at the absorption line centre and its amplitude is proportional to the concentration of the target gas. The signal suffers from less noise and has a flat baseline which makes it the ideal signal choice to be measured in most WMS schemes for low level absorption measurements. The $1 \mathrm{~F}$ signal is antisymmetric and has a dc offset at the line centre which is proportional to the laser output power. Hence it is susceptible to laser power fluctuations and may not be ideal for frequency stabilisation unless the dc offset remains a constant. On the other hand, the $3 \mathrm{~F}$ signal suffers from no such offset and has been employed in some WMS systems for frequency stabilisation of the laser output. A more detailed description of WMS including theoretical models is available elsewhere in the literature ${ }^{3,5,10}$.

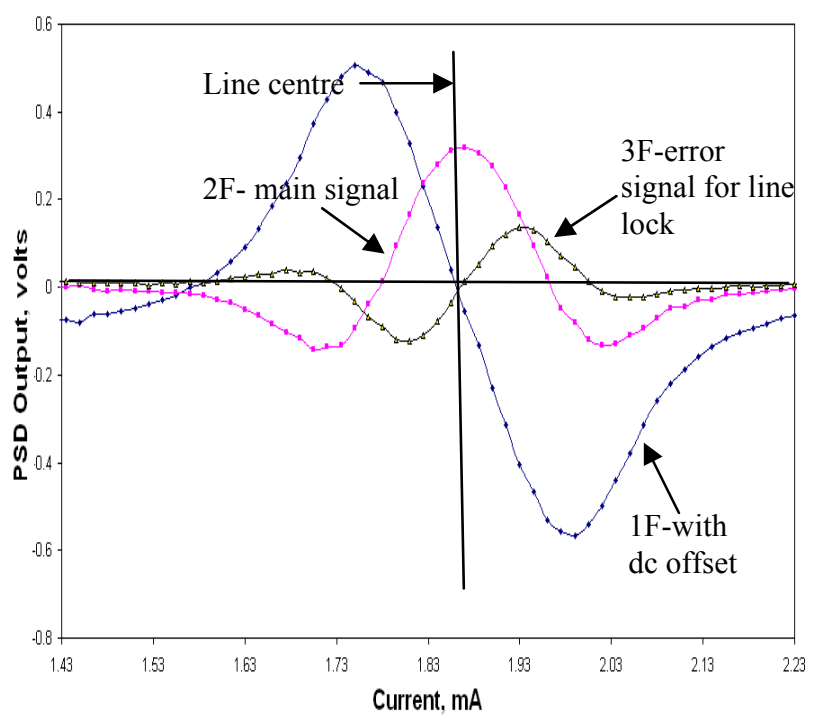

Figure 3. Different harmonics generated in a typical WMS system. These curves were obtained for $100 \% \mathrm{CH}_{4}$ in a $10 \mathrm{~cm}$ gas cell as the laser injection current was swept across the line centre at $1651 \mathrm{~nm}$.

\section{PROPOSED SCHEME AND EXPERIMENTAL DESIGN}

\subsection{Block Diagram}

A block diagram of the proposed scheme is shown in figure 4. The inbuilt thermoelectric cooler (TEC) on the VCSEL was used to precisely control the temperature of the VCSEL while the injection current was slowly ramped at a few $\mathrm{Hz}$ using an external digitally generated saw tooth signal. By using appropriate VCSEL temperature and current settings, the methane gas peak was obtained in the middle of the current sweep. A low amplitude sine wave was then superimposed on the slow ramp for wavelength modulation at a frequency, $\omega_{m}$, which was initially kept at $6.5 \mathrm{kHz}$. The laser emission was made to pass through an absorption cell containing methane and the transmitted light was captured using an InGaAs photodiode. The DC signal from the photodiode was then fed to a PSD with an output time constant of $2 \mathrm{~ms}$ for recovery of the $2 \mathrm{~F}$ signal. A 16 bit DAQ card from National Instruments was used with LABVIEW for data acquisition during lab trials.

\subsection{Optimisation of $2 F$ signal}

To maximise the recovered $2 \mathrm{~F}$ signal, some optimisation procedures were followed which are presented here. Sensitivity of the WMS scheme depends on the modulation depth used which is represented by the modulation index, $m$. It is defined as the ratio of modulation amplitude, $v_{\mathrm{m}}$, to the half width half maximum (HWHM) of the Lorentzian absorption feature, $v_{\mathrm{L}}$, i.e.

$$
m=\frac{v_{m}}{v_{L}}
$$




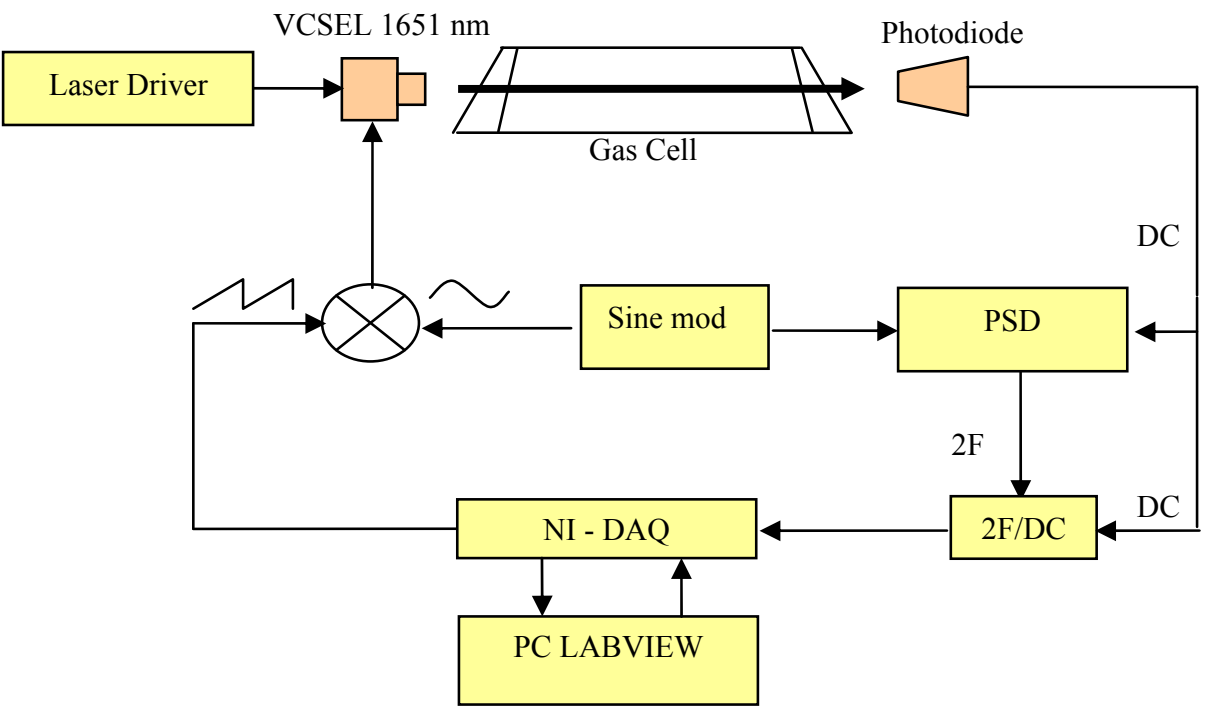

Figure 4. Schematic of the proposed WMS setup. PSD, phase sensitive detector; NI-DAQ, National Instruments data acquisition card.

For maximising the $2 \mathrm{~F}$ output of a WMS system, an optimum value of 2.2 should be chosen for $m$, implying that the amplitude of modulation should be slightly greater than the FWHM of the absorption line. This is derived from analytical expressions used to define a modulated Lorentzian absorption line shape as given by Arndt ${ }^{11}$. Experimental test results as shown in figure 5 exhibit very good correlation with the established theory. Optimised modulation amplitudes were therefore used to maximise the $2 \mathrm{~F}$ output of the experimental WMS system.

It can be deduced from equation 2 that at ambient or higher pressures large modulation amplitudes are required to effectively scan across a pressure broadened absorption feature. A higher modulation index, although maximising the $2 \mathrm{~F}$ signal, also has the undesired effect of maximising residual amplitude modulation (RAM). Many studies have been carried out in the past ${ }^{12-14}$ to describe and analyse the effect of RAM and only a brief insight into its contribution to the $2 \mathrm{~F}$ signal is provided here. When the laser injection current is modulated at a frequency $\omega_{m}$, it also results in an inherent but unwanted RAM or intensity modulation (IM) of the emitted power. However, the wavelength or frequency modulation (FM) achieved, which is a result of a thermal process within the laser chip, lags behind IM due to the thermal inertia of the laser by an angle $\Phi_{m}$. The $2 \mathrm{~F}$ signal generated can be analytically represented ${ }^{12}$ as

$$
i_{2 f}(t)=-\frac{1}{2} i_{0}\left[\frac{a^{(2)} \delta v^{2}}{2 !}+\frac{a^{(4)} \delta v^{4}}{4 !}+\ldots\right] \cos \left(2 \omega_{m} t-2 \phi_{m}\right)-\frac{1}{2} \delta i a^{(1)} \delta v \cos \left(2 \omega_{m} t-\phi_{m}\right)+\ldots
$$

where $i_{0}$ is the average intensity at the detector, $\delta v$ and $\delta i$ represent modulation in terms of frequency and laser current and $a^{(n)}$ is the $\mathrm{n}^{\text {th }}$ derivative of the absorption line shape. It can thus be seen that at a lower modulation index, $\delta v$ and $\delta i$ have smaller values and hence majority of the contribution to the $2 \mathrm{~F}$ signal is from the second order derivative of the absorption line shape. However, at larger modulation amplitudes which are necessary at ambient pressures, contribution from the first derivative of the line shape becomes significant due to the $\delta i a^{(l)}$ term which is a direct consequence of the IM described earlier. Moreover, this IM contribution to the $2 \mathrm{~F}$ signal is phase shifted by $\Phi_{m}$ whereas the desired FM contribution to the $2 \mathrm{~F}$ signal has a phase shift of $2 \Phi_{m}$ as seen in the equation. To maximise the FM contribution and null the IM effect, the reference phase on the PSD has to be set to $2 \Phi_{m}$. This however may also result in a change in the overall $2 \mathrm{~F}$ line shape. 


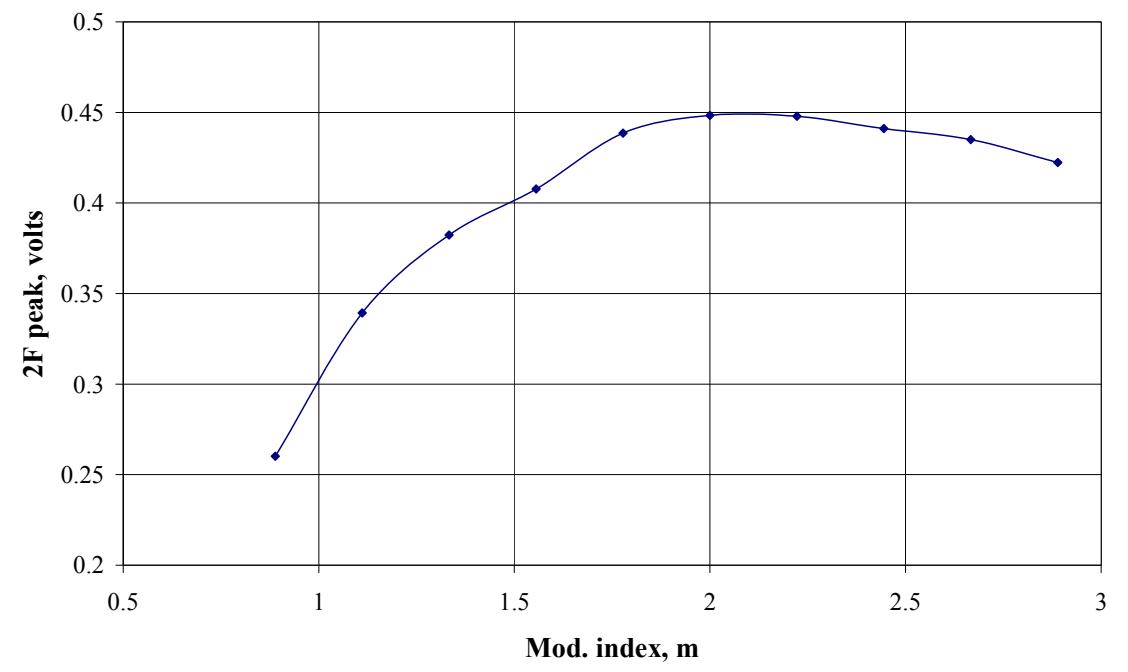

Figure 5. Experimental data showing variation of $2 \mathrm{~F}$ peak values with modulation index, $\mathrm{m}$. Plot shows a maximum for $2 \mathrm{~F}$ peak value when $\mathrm{m}$ equals 2.2 as predicted by analytical models.

The optimised $2 \mathrm{~F}$ signals were then normalised by the average photodiode dc output so that laser output power fluctuations are compensated for which allows for a direct comparison between various test results carried out under varying laser drive settings. This method of normalisation was preferred over the standard $2 \mathrm{~F} / 1 \mathrm{~F}$ normalisation scheme since normalisation using $1 \mathrm{~F}$ relies on the exact relation between laser output power and $1 \mathrm{~F}$ signal level, which may change with laser ageing ${ }^{4}$.

\section{EXPERIMENTAL PROGRAMME}

\subsection{Test results}

The most common form of performance limitation encountered in a typical WMS system is usually from optical interference fringes. Initial experiments were therefore carried out to conduct a comparative study of different optical layouts and the resultant optical fringes on the $2 \mathrm{~F}$ signal. There are different types of optical fringes that arise from different sources and their distinction is not always very clear. Standard FabryPerot etalons formed between parallel optical surfaces produce fringes that have nearly the same width as the gas line being scanned and their amplitudes may be big enough to obscure small absorption features thereby limiting detection sensitivity. Fringes due to self mixing interference may also arise due to external feedback into the laser diode from reflective surfaces along the optical path ${ }^{15}$. By measuring the free spectral range (FSR) of these fringes, the corresponding optical distance can be calculated which will help to identify their point of origin. Hence optimum design and alignment of the various optical components is critical in developing a low noise optical system.

Figure 6 shows the effect of optical fringes observed in an experimental $2 \mathrm{~F}$ background scan when a $10 \mathrm{~cm}$ long gas cell was used without any windows. The FSR for the high frequency fringes correspond to an optical cavity length of $\sim 10 \mathrm{~cm}$ which is the etalon created between the windows on the detector and the VCSEL. The effect of self mixing interference is also clearly visible in the figure in the form of a slowly modulating envelope signal. These effects were reduced by almost $80 \%$ by sufficiently angling the detector with respect to the source. 


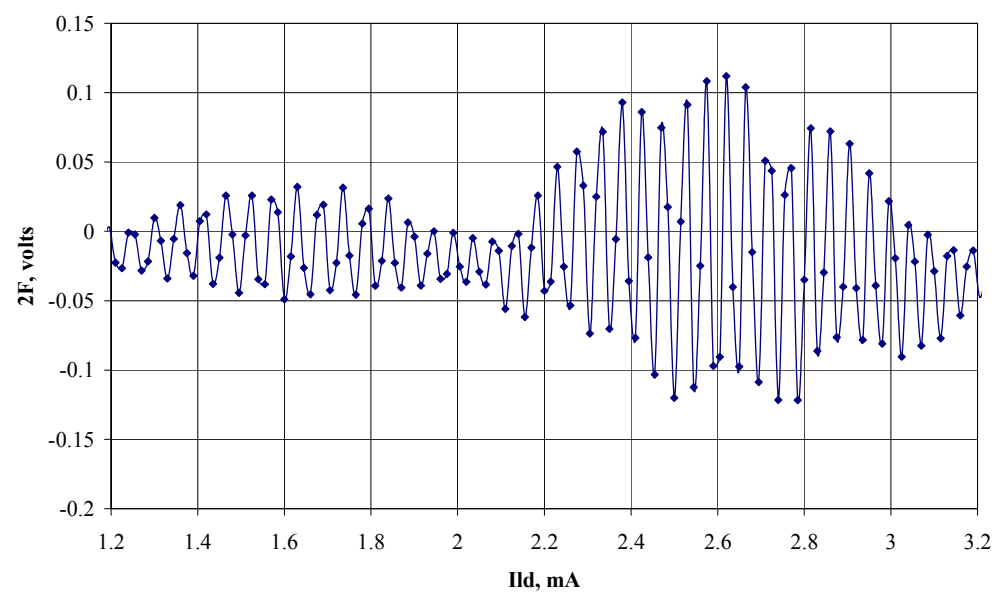

Figure 6. A 2F background scan using a $10 \mathrm{~cm}$ pathlength. The effect of etalon fringes and self mixing interference fringes are clearly visible.

Further tests were carried out to evaluate various optical cells made with different materials and diameters, the effect of wedged optical windows and the use of apertures. Based on the conclusions drawn, a demonstrator was built and extensive performance evaluation and gas based tests were carried out to identify problems and possible areas of improvement. Methane gas mixtures containing $500 \mathrm{ppm}, 100 \mathrm{ppm}$ and $50 \mathrm{ppm}$ were measured using the demonstrator. Advanced data processing techniques were implemented within LABVIEW to obtain the final processed $2 \mathrm{~F}$ curves as shown in figure 7.

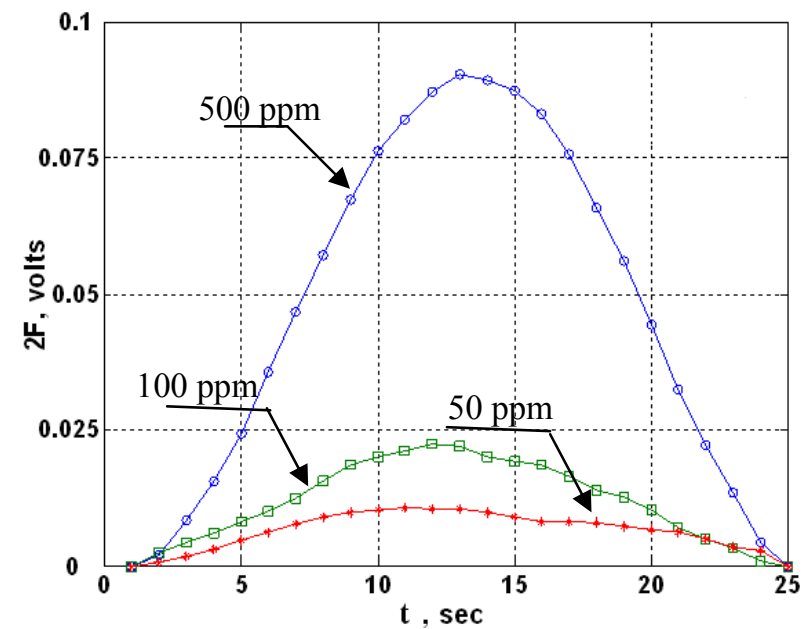

Figure 7. Corrected $2 \mathrm{~F}$ scans after data processing in LABVIEW.

Although better short term performance was possible, long term drift effects from background fluctuations were a limiting factor for the current application. The presence of this non-constant $2 \mathrm{~F}$ background signal has been widely reported in literature and various reasons have been attributed to it. These include non-linearity of power-current characteristics of the $\mathrm{VCSEL}^{14}$, high modulation depth requirement for measurements at ambient pressures ${ }^{16}$, contribution of IM-FM phase shift leading to reduced $2 \mathrm{~F}$ signa ${ }^{14}$, possible noise from laser side modes ${ }^{17}$ and intrinsic laser noise. A simple and practical solution to improve the signal to noise ratio (SNR) is to increase the effective absorption path length in order to raise gas signal amplitude above background levels. This must be done ensuring the resultant increase in the volume to be purged does not adversely affect the instrument response time, which is a critical factor for the intended field application. This was tested by employing a $20 \mathrm{~cm}$ long double pass arrangement as shown in figure 8 giving an effective path length of $40 \mathrm{~cm}$. A spherical concave mirror was used to reflect the laser emission on 
to the photodiode. Using this arrangement a $5 \mathrm{ppm}$ limit of detection (LOD) was achieved, equivalent to $10^{-5}$ in absorbance, with adequate long term stability.

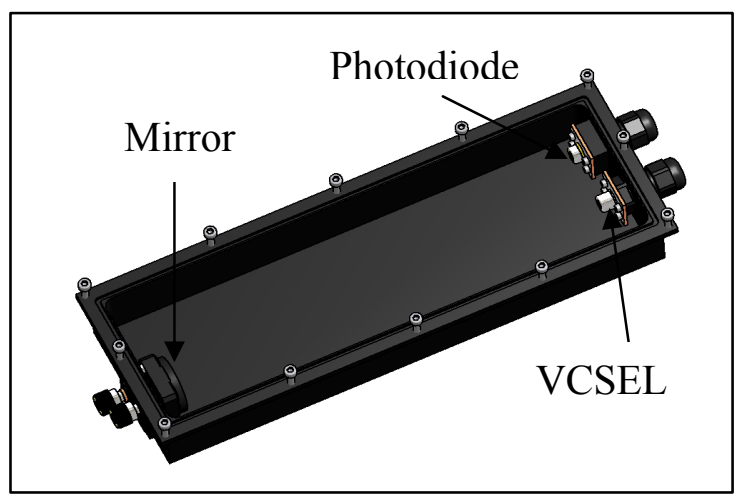

Figure 8. Double pass arrangement used to achieve $5 \mathrm{ppm}$ LOD using a $40 \mathrm{~cm}$ effective path length.

\subsection{Data Processing}

The $2 \mathrm{~F}$ curves obtained after the optimisation and normalisation procedures mentioned above were then processed to improve data quality. A curve fitting procedure using the Levenberg-Marquardt non-linear least squares algorithm was implemented in $\mathrm{C}++$ to precisely calculate peak $2 \mathrm{~F}$ values and hence the gas concentration. This procedure was based on the analytical model of a Lorentzian gas absorption line shape as given by $\operatorname{Arndt}^{11}$. Adequate compensation schemes for both temperature and pressure were also incorporated into the system to obtain corrected gas concentration values. Figure 9 shows the effect of the curve fit on the raw $2 \mathrm{~F}$ values obtained for $50 \mathrm{ppm} \mathrm{CH}_{4}$. Using a standard $\mathrm{PC}, 400$ data points were processed within $200 \mathrm{~ms}$, which was adequately fast for the field prototype.

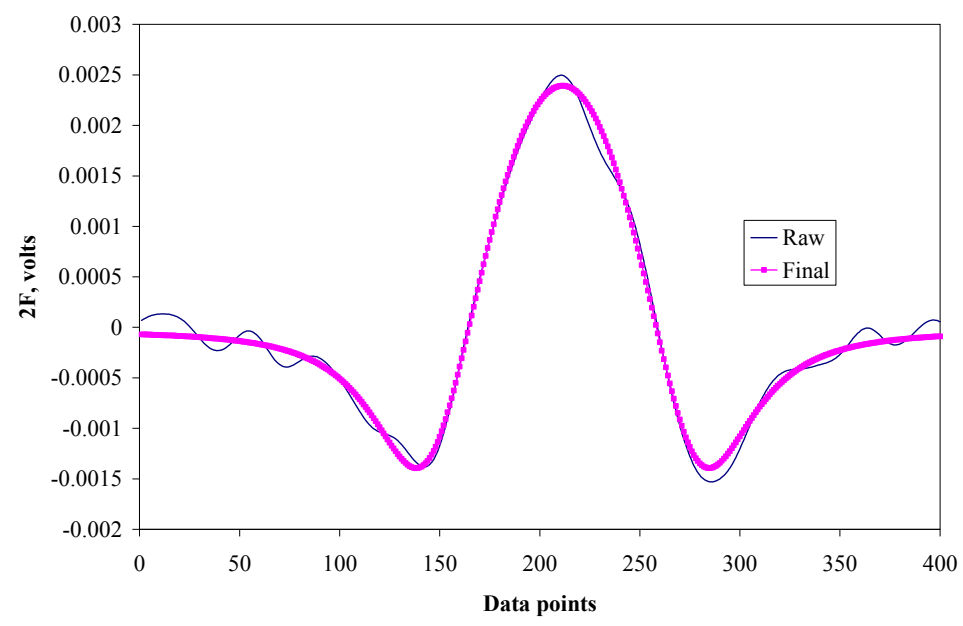

Figure 9. Experimental $2 \mathrm{~F}$ scans obtained for $50 \mathrm{ppm} \mathrm{CH}_{4}$ concentration over $40 \mathrm{~cm}$. Raw refers to the unprocessed $2 \mathrm{~F}$ data. Final was obtained after curve fit.

\subsection{Field Prototype Instrument}

This section gives details about the field deployable prototype instrument that is currently undergoing performance and calibration tests in the lab. The prototype should be capable of measuring close to background atmospheric $\mathrm{CH}_{4}$ levels of $1.6 \mathrm{ppm}$. In order to gain the required sensitivity and SNR, a multi-pass Herriot cell with a design pathlength of $4 \mathrm{~m}$ was used. An aspheric collimating lens was used with the VCSEL and special mounts were designed to hold the VCSEL and photodiode assembly in place. The overall optical assembly was designed to be rugged and to minimise any stray or unwanted reflections that may later appear as a source of interference fringes. A new embedded processor board was 
used in conjunction with an ADC/DAC card that offered the required 16 bit resolution. Optimum values for the modulation frequency and the ramp rate were chosen.

The field prototype being built will form part of the Automated Extraction Monitoring System (AEMS) ${ }^{18}$ that Geotechnical Instruments currently offers as a standard fixed system monitoring solution. This system has an in-built gas conditioning unit to remove harmful gases and water vapour present in the input mix. A simplified schematic of the AEMS gas conditioning unit and data communications is shown in figure 10.

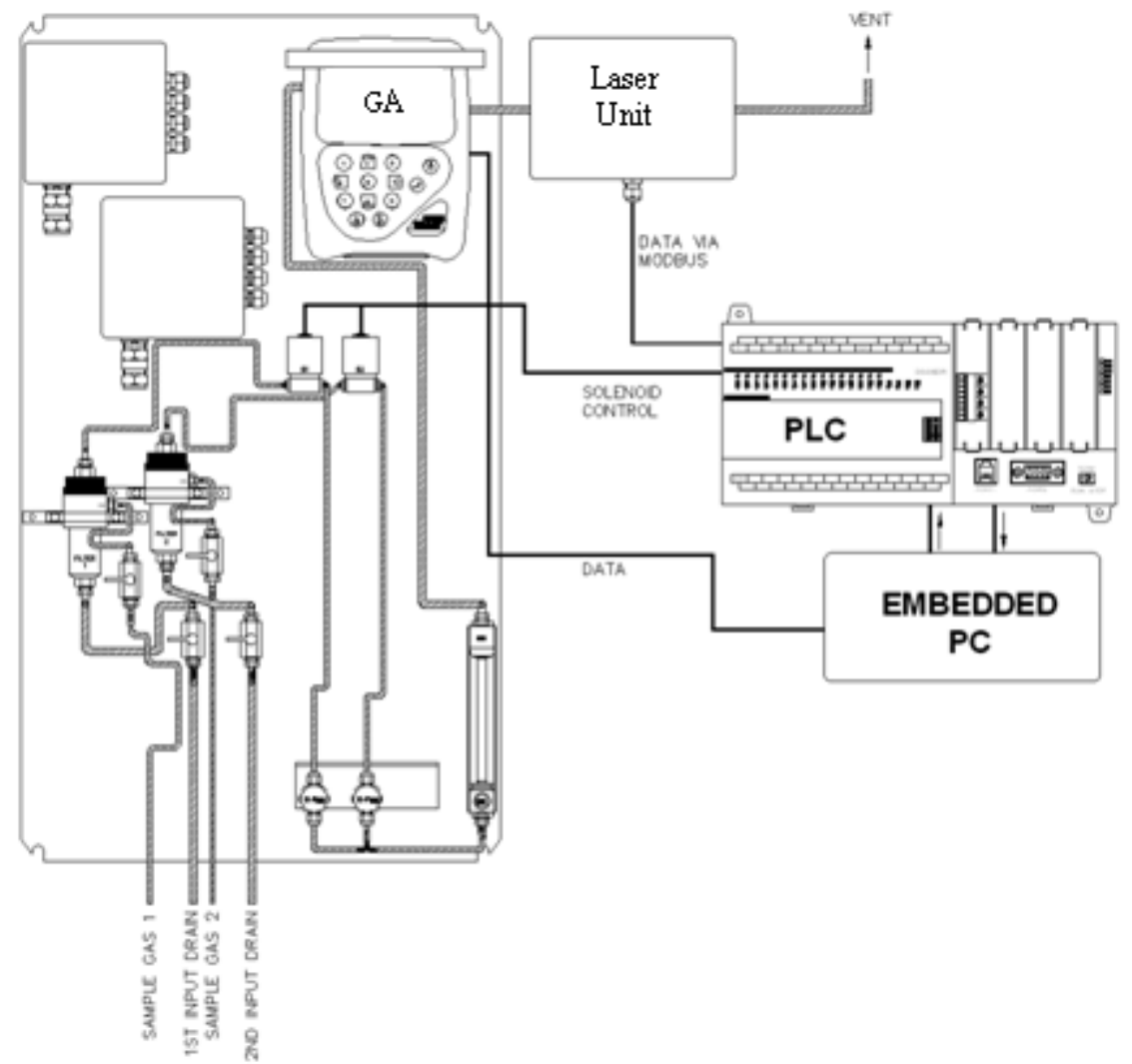

Figure 10. Simplified schematic of AEMS gas conditioning and communications layout. GA, main gas analyser; PLC, programmable logic controller.

The main GA gas analyser measures gases such as $\mathrm{CO}_{2}, \mathrm{O}_{2}$ and $\mathrm{H}_{2} \mathrm{~S}$ and the data is sent to the embedded PC. Data from the embedded board on the laser unit is periodically sent to a programmable logic controller (PLC) within the AEMS via MODBUS protocols. The embedded PC on the AEMS then periodically requests data from the PLC location and the data can either be displayed in situ or sent to a remote location via the web for storage and analysis. This facilitates a completely remote and automated system for monitoring the low ppm levels of residual methane.

\section{CONCLUSION}

Measurement of low ppm levels of methane in an industrial environment has gained commercial significance recently as a means of earning carbon credits. This paper discusses the various steps taken and design challenges faced while developing a field deployable commercial instrument that is robust in design and reliable in its operation. A WMS scheme with second harmonic detection was employed and curve fitting procedures were used to improve sensitivity. Various optical designs were evaluated to limit the effect of interference fringes and a LOD of $5 \mathrm{ppm}$ was achieved. The laser unit will form part of an automated system that provides an ideal solution for remote on-site monitoring of gases. The field prototype is currently undergoing lab tests and will then be deployed for field trials. 


\section{ACKNOWLEDGEMENTS}

The authors would like to gratefully acknowledge the financial support received from the Technology Strategy Board (TSB) for carrying out this work. This project was part funded by the TSB under the Knowledge Transfer Partnership programme (KTP 6825).

\section{REFERENCES}

1. http://www.mass.gov/dep/recycle/laws/lfgasapp.pdf

2. http://unfccc.int/resource/docs/convkp/kpeng.pdf

3. Fernholz, T., Teichert, H. and Ebert, V., "Digital, phase-sensitive detection for in situ diode-laser spectroscopy under rapidly changing transmission conditions," Appl. Phys. B, 75, 229-236 (2002).

4. Chen, J., Hangauer, A., Strozda, R. and Amann, M. C., "Laser spectroscopic oxygen sensor using diffuse reflector based optical cell and advanced signal processing,” Appl. Phys. B, 100(2), 417-425 (2010).

5. Chao, X., Jeffries, J. B. and Hanson, R. K., "Absorption sensor for CO in combustion gases using $2.3 \mu \mathrm{m}$ tunable diode lasers," Meas. Sci. Technol., 20, 1-9 (2009).

6. Rieker, G. B., Li, H., Liu, X., Jeffries, J. B., Hanson, R. K., Allen, M. G., Wehe, S. D., Mulhall, P. A. and Kindle, H. S., "A diode laser sensor for rapid, sensitive measurements of gas temperature and water vapour concentration at high temperatures and pressures," Meas. Sci. Technol., 18, 1195-1204 (2007).

7. Zhu, X. and Cassidy, D. T., "Modulation spectroscopy with a semiconductor diode laser by injection-current modulation,” J. Opt. Soc. Am. B., 14, 1945-1950 (1997).

8. D' Amato, F., Mazzinghi, P. and Castagnoli, F., "Methane analyzer based on TDL's for measurements in the lower stratosphere: design and laboratory tests," Appl. Phys. B, 75, 195-202 (2002).

9. Rothman, L. S. et al., "The HITRAN 2004 molecular spectroscopic database,” J. Quant. Spect. Rad. Transf., 96, 139-204 (2005).

10. Reid, J. and Labrie, D., "Second harmonic detection with tunable diode lasers - comparison of experiment and theory," Appl. Phys. B, 26, 203-210 (1981).

11. Arndt, R., "Analytical line shapes for Lorentzian signals broadened by modulation,” J. Appl. Phys., 36, 2522-2524 (1965).

12. Schilt, S. and Thevenaz, L., "Experimental method based in wavelength modulation spectroscopy for the characterization of semiconductor lasers under direct modulation," Appl. Opt., 43(22), 4446-4452 (2004).

13. Schilt, S., Thevenaz, L. and Robert, P., "Wavelength modulation spectroscopy: combined frequency and intensity laser modulation," Appl. Opt., 42(33), 6728-6738 (2003).

14. Engelbrecht, R., "A compact NIR fiber - optic diode laser spectrometer for $\mathrm{CO}$ and $\mathrm{CO}_{2}$ : analysis of observed $2 \mathrm{f}$ wavelength modulation spectroscopy line shapes," Spectrochimica Acta A, 60, 3291-3298 (2004).

15. Masiyano, D., Hodgkinson, J., Schilt, S. and Tatam, R. P., "Self mixing interference effects in tunable diode laser absorption spectroscopy," Appl. Phys. B, 96, 863-874 (2009).

16. Zhu, X. and Cassidy, D. T., "Electronics subtractor for trace-gas detection with InGaAsP diode laser," Appl. Opt., 34(36), 8303-8308 (1995).

17. Ventrudo, B. F. and Cassidy, D. T., "Operating characteristics of a tunable diode laser absorption spectrometer using short-external-cavity and DFB laser diodes,” Appl. Opt., 29(33), 5007-5013 (1990).

18. http://www.geotech.co.uk/aems.php 